\title{
Markus Wien
}

\section{Die bulgarische Monarchie: Politisch motivierte Revision eines Geschichtsbildes in der Transformationsgesellschaft}

Das vorsozialistische Bulgarien, das als Nationalstaat neuzeitlicher Prägung im Jahre 1878 als Folge des vorausgegangenen russisch-türkischen Krieges gegründet worden war, hatte von Beginn an eine monarchische Staatsform und behielt diese unter gewissen Veränderungen bis 1946 bei. Der erste Bruch ereignete sich bereits 1886 in Form eines Wechsels der Dynastie, als Fürst Alexander v. Battenberg nach dem bulgarisch-serbischen Krieg die außenpolitische Unterstützung Rußlands verlor und unter dem Druck des Zarenreiches abdanken mußte. Ihm folgte das Haus von Sachsen, Coburg und Gotha, das bis 1946 drei Monarchen stellte: Ferdinand I. (1886-1918), Boris III. (1918-1943) und für kurze Zeit den minderjährigen Simeon II. (1943-1946). Die zweite bedeutsame Veränderung vollzog sich 1908 mit der endgültigen Abschüttelung der osmanischen Tributhoheit und der Erhebung des bisherigen Fürstentums Bulgarien zum Zarenreich - Ferdinand hieß fortan „Zar der Bulgaren“. Dies blieb bis 1946 der offizielle Titel des Staatsoberhauptes, wenn auch nach dem 9. September 1944 die Kommunisten das Land beherrschten und die Monarchie mit dem kleinen, durch einen dreiköpfigen Regentenrat vertretenen Simeon bis 1946 im Grunde nur noch eine Institution „in Abwicklung" war. Ihre endgültige Abschaffung erfolgte am 8. September 1946 durch ein manipuliertes Referendum, das Bulgarien in eine "Volksrepublik" umwandelte. Simeon mußte mit seinem Gefolge das Land verlassen, dankte jedoch nicht formell ab, sondern führte als Exilmonarch seinen Herrschertitel weiter ${ }^{1}$.

In den Jahren des Sozialismus bis 1989 war allerdings an eine Rückkehr in seine Heimat nicht zu denken - von der Übernahme der Funktionen eines Staatsoberhauptes ganz zu schweigen. Zu eindeutig lehnten die Kommunisten die Monarchie als reaktionär und feudalistisch $\mathrm{ab}$ und damit auch ihre Repräsentanten. Dementsprechend war ihnen daran gelegen, in der Öffentlichkeit jede Form der positiven Erinnerung daran zu verhindern, indem sie ein Geschichtsbild der ersten Hälfte des 20. Jahrhunderts erzeugten, das die Monarchie als Ursache oder

1 Allgemein zur bulgarischen Geschichte vgl. u.a. R. J. Crampton, Historical Foundations, in: K.-D. Grothusen (Hrsg.), Südosteuropa-Handbuch, Bd. VI. Bulgarien (Göttingen 1990) 27-55. 
zumindest als wichtige Voraussetzung für alle negativen Entwicklungen in Bulgarien seit 1878 darstellte. Griffige Bezeichnungen, wie etwa „Abenteurertum“ für die Beteiligung Bulgariens an den Balkankriegen und dem Ersten Weltkrieg unter Zar Ferdinand oder "Monarchofaschismus“ für die autoritäre Regierungsform zur Zeit Boris' III., wurden gewählt, um die Epoche vor 1944 als eine dunkle, aber überwundene Vergangenheit im Bewußtsein der Menschen zu verankern².

Nach der „Wende“ 1989/90 und der Beseitigung des kommunistischen Meinungsmonopols ergab sich relativ unvermittelt die Möglichkeit einer historischen Neubewertung der bulgarischen Monarchie. Vor allem Journalisten und populärbis pseudowissenschaftlich arbeitende Autoren setzten sich an die Spitze einer Bewegung, die die Monarchie gelegentlich verklärte, auf jeden Fall aber als positives Gegenbild zur Ein-Partei-Diktatur der sozialistischen Zeit präsentierte. Zu Beginn der 90er Jahre war dies in erster Linie Ausdruck des Gebrauchs der neuen Meinungsfreiheit und weniger einer politischen Gesinnung. Folge davon war allerdings, daß die Geschichtswissenschaft innerhalb dieses Meinungsbildungsprozesses ins Hintertreffen geriet, hatten doch die meisten ihrer Vertreter jahrelang selbst zur Formung des von der Staatsführung gewünschten negativen Bildes von der Monarchie beigetragen ${ }^{3}$. Auch eine Bewegung zur Wiedereinführung der Monarchie bildete sich in Bulgarien in Form einer Partei. Sie blieb allerdings ohne nennenswerten politischen Einfluß; die einzige politische Entscheidung in ihrem Sinne war Mitte der 90er Jahre die Wiedereinführung des königlichen Staatswappens ${ }^{4}$.

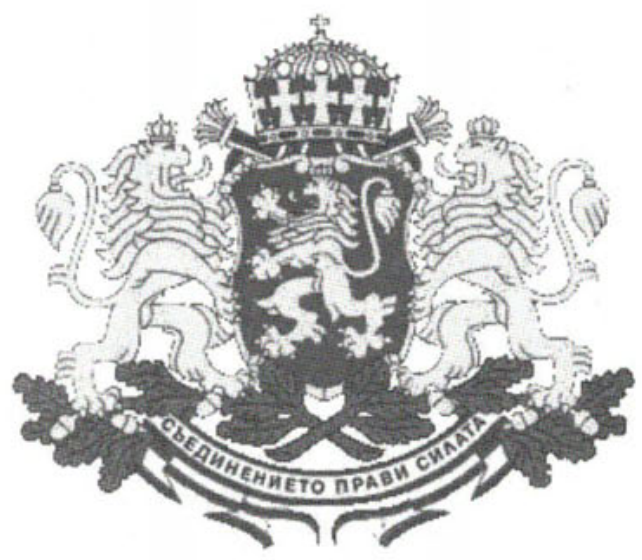

2 Vgl. B. Koen, Die monarchofaschistische Oberschicht, Hitlerdeutschland und die Judenfrage, in: Bulgarische Akademie der Wissenschaften. Institut für Geschichte (Hrsg.), Bulgarisch-deutsche Beziehungen und Verbindungen. Bd. 4 (Sofia 1989) 20-41, hier 20-22.

${ }^{3}$ Vorreiter dieser außerakademischen Historiographie sind etwa der medienpräsente Autor Božidar Dimitrov sowie das Verlagshaus "TANGRA TanNakRa“, nach einer proto-bulgarischen Gottheit benannt. Vgl. zur bulgarischen „Para-Historiographie“ I. Iliev, On the history of inventing Bulgarian history, IWM Working Paper 5 (Wien 2000) 13.

${ }_{4}$ Zur Partei: >http://www.ewis.de/bgppart.html<, vgl. auch www.election.bg, Staatswappen: vgl. > http://flagspot.net/flags/bg.html<. 
Auftrieb erhielt die monarchische Bewegung allerdings ab 1996 durch die wiederholten Besuche, die Simeon II. von seinem spanischen Exil aus dem Lande abstattete. Eine politische Perspektive eröffnete sich ihm allerdings erst um die Jahrtausendwende, als sich in Bulgarien zunehmend Enttäuschung über alle etablierten Parteien und deren Repräsentanten breit machte und man nach völlig neuen Kräften zu suchen begann. Diejenigen, die in dieser Situation für die Wiedereinführung der Monarchie eintraten, verkannten allerdings gänzlich, daß diese Staatsform zumindest dort, wo sie in Europa noch existierte, durch und durch parlamentarisiert war, daß also die gekrönten Staatsoberhäupter keinerlei politische Macht, sondern nur repräsentative Funktionen ausübten. Die Rückkehr eines Zaren, der aktiv in die Politik eingegriffen hätte, wäre also nur um den Preis der Demokratie und damit der angestrebten Integration in die euro-atlantischen Strukturen möglich gewesen. Eine parlamentarische Monarchie hätte hingegen wider den Erwartungen ihrer Anhänger eine Veränderung der politischen Landschaft nicht notwendigerweise herbeigeführt ${ }^{5}$.

Ohnehin hat sich hierfür in Bulgarien bis heute keine Mehrheit gefunden. Simeon gelang es aber, im Volk so viele Sympathien für sich zu wecken, daß er im Frühjahr 2001 den Schritt auf die politische Bühne des Landes wagen konnte: Er nahm an den Parlamentswahlen vom 17. Juni des Jahres teil und errang mit rund 43 Prozent, die auf seine "Nationale Bewegung Simeon II.“ (NDSV) entfielen, einen überwältigenden Wahlsieg. Paradoxerweise war er nun gezwungen, Ministerpräsident zu werden, also ein republikanisches Staatsamt anzunehmen dabei ist nicht anzunehmen, daß er dies aktiv angestrebt hat, da er noch nach den Wahlen lange zögerte, bevor er sich zu diesem Schritt entschloß. Denn nun stand er, der als Zar nie abgedankt hatte, in der protokollarischen Hierarchie unter dem Staatspräsidenten, dessen Stellvertreter und dem Parlamentspräsidenten ${ }^{6}$.

Trotzdem wäre sein Wahlerfolg kaum erklärbar gewesen, wenn sich nicht die Einstellung der bulgarischen Öffentlichkeit zur Monarchie seit 1989 grundlegend verändert hätte - auch wenn noch eine Reihe anderer Gründe für seine Wahl eine Rolle gespielt hat. Diese Einstellung, die in Simeon ihren lebendigen Kristallisationspunkt gefunden hat, bezieht sich allerdings beim Rückblick auf die vorsozialistische Zeit in erster Linie auf die Persönlichkeiten der Monarchen und weniger auf die Institution der Monarchie als solche, d.h. auf ihre verfassungsrechtliche Bedeutung. Dies gilt nicht nur seit 1989, sondern galt im allgemeinen auch schon davor.

5 M. Wien, ${ }_{n} \mathrm{Ab}$ heute ist Bulgarien nicht mehr dasselbe Land“. Die Parlamentswahlen vom 17. Juni 2001, in: Südosteuropa. Zeitschrift für Gegenwartsforschung 4 (2001) 13-32, hier 13. 6 Ebd. 


\section{I. „Abenteurertum“ und „Monarchofaschismus“}

Auf dem Gebiet der historischen Einordnung und Bewertung der bulgarischen Monarchie äußerte sich die sozialistische Geschichtsforschung des Landes am prononciertesten zur Regierungszeit und zur Person Boris' III. Seine Vorgänger auf dem Thron fanden dagegen vergleichsweise wenig Beachtung, am wenigsten der erste Fürst von Bulgarien, Alexander von Battenberg. Die stets auch aus einem national-bulgarischen Blickwinkel urteilende offizielle Historiographie konnte allerdings nicht umhin, ihm wegen der unter seiner Regentschaft vollzogenen Vereinigung Ostrumeliens mit dem Fürstentum 1885 im Zuge des bulgarisch-serbischen Krieges immerhin auch ein gewisses $\mathrm{Ma} ß$ an Anerkennung entgegen zu bringen. Seine fortgesetzten Versuche zur Aushöhlung der von ihm von Beginn an innerlich abgelehnten, in der Verfassung von Tărnovo festgeschriebenen parlamentarisch-demokratischen Staatsform, die in der Suspendierung der Verfassung zwischen 1881 und 1883 gipfelten, brachten ihm jedoch nach 1944 insgesamt den Ruf eines reaktionären, feudalistisch eingestellten Autokraten ein? ${ }^{7}$. Allerdings wird dieses Urteil von der postsozialistischen Geschichtsschreibung auch heute noch weitgehend geteilt, wobei nun aber seine nationalpolitischen Erfolge im Vordergrund stehen. Das Scheitern seines autokratischen innenpolitischen Kurses wird hingegen in erster Linie als Ausdruck des demokratischen Widerstandsgeistes des bulgarischen Volkes angesehen ${ }^{8}$. Immerhin aber wird Fürst Alexander heute insoweit als "positive" Gestalt in der modernen bulgarischen Geschichte gewürdigt, als beispielsweise der Sofioter Alexander-von-Battenberg-Platz vor dem ehemaligen Königsschloß seinen alten Namen zurück erhalten hat. Hier zeigt sich indes das bereits erwähnte Phänomen einer Rückbesinnung auf historische Überlieferungen, das zunächst weniger auf einer bewußten Hinwendung zu denselben beruhte, als vielmehr auf dem Bestreben, sich möglichst vieler Spuren der jüngsten kommunistischen Vergangenheit zu entledigen'.

Als im Endergebnis gescheiterter Monarch ist Zar Ferdinand sowohl in die sozialistische als auch in die postsozialistische Historiographie wie auch in das öffentliche Geschichtsbewußtsein eingegangen ${ }^{10}$. Der entscheidende Grund dafür ist naheliegenderweise die Niederlage Bulgariens im Ersten Weltkrieg, die die Abdankung Ferdinands und seine Rückkehr an den deutschen Stammsitz seines Geschlechts zur Folge hatte. Zwar schlug für ihn positiv zu Buche, daß unter seiner Regentschaft Bulgarien die vollständige staatliche Souveränität erlangte, indem es sich im Zuge der Jungtürkischen Revolution von den Tributzahlungen an

7 Vgl. hierzu: J. Geševa, Režimăt na pălnomoštijata (1881-1881) - svoeobrazna forma na upravlenie na dăržavata, in: Bălgarska akademija na naukite. Institut po istorija (Hrsg.), 120 godini izpǎlnitelna vlast v Bălgarija. Konferenzband (Sofia 1999) 91-103.

8 Ebd.

9 Zuvor hieß er "Platz des 9. September“ nach dem Datum der kommunistischen Machtübernahme 1944, vgl. >www.sofiaecho.com/sofsight05.php<.

10 Bulgaria.com. Außerdem: $M$. Petrov, Nacionalnijat văpros v politikata na bălgarskite pravitelstva (1879-1919), in: 120 godini 128-147. 
Konstantinopel befreite und zum Zarenreich avancierte, den größten Posten in Ferdinands Regierungsbilanz nimmt jedoch die letztlich in der „nationalen Katastrophe“ resultierende Teilnahme Bulgariens an den drei Kriegen zwischen 1912 und 1918 ein". Herrscht über den „katastrophalen“ Charakter dieses Geschehens und der ihm zugrunde liegenden politischen Entscheidungen allgemeine Einigkeit, so sind bezüglich der Bewertung ihrer Motive durchaus Wandlungen im Rahmen des Systemwechsels nach 1989 festzustellen. Politisches und militärisches "Abenteurertum" wird Ferdinand in diesem Zusammenhang von der sozialistischen Historiographie zum Vorwurf gemacht. Entsprechend den grundlegenden marxistischen Bewertungsmustern wird dahingehend argumentiert, daß der Monarch, im Dienste bürgerlicher Klasseninteressen stehend, versucht habe, mittels des Krieges die inneren Widersprüche des Kapitalismus zu überdecken, das werktätige Volk zu disziplinieren und den Internationalismus der Proletarier zu unterdrücken, indem er sie gegen ihre Klassengenossen in anderen Ländern hetzte. Insgesamt habe das Regime Ferdinands sich zum Erfüllungsgehilfen kapitalistischer und, durch das Bündnis mit den Mittelmächten, auch imperialistischer Zielsetzungen gemacht und somit den Interessen des „Volkes“ zuwidergehandelt ${ }^{12}$.

Seit dem Ende der kommunistischen Herrschaft in Bulgarien sind Tendenzen zu einer differenzierteren Beurteilung des Ferdinandschen Regiments spürbar. Besonders für seine jeweiligen Entscheidungen zur Teilnahme an den drei Kriegen bringen sowohl wissenschaftliche Geschichtsforschung als auch Publizistik zunehmend Verständnis auf. Rückgriffe auf Bewertungsmuster, die noch aus der Zwischenkriegszeit stammen, sind vielfach kennzeichnend für diese Strömungen. Ein prominentes Beispiel hierfür ist etwa der eindeutig nationalistisch eingestellte Autor Božidar Dimitrov, dessen Werke in der bulgarischen Öffentlichkeit weite Verbreitung finden und daher auch einen nicht unerheblichen Einfluß auf das Geschichtsbild des breiten Publikums haben. Auch zahlreiche Auftritte im Fernsehen tragen zur Popularität Dimitrovs bei. Seiner Terminologie zufolge handelt es sich bei den Balkankriegen und dem Ersten Weltkrieg nicht mehr um militärische Abenteuer zur Durchsetzung von Klasseninteressen, sondern um nationale Einigungskriege, die diejenigen Bulgaren, die noch unter fremder, d.h. osmanischer Herrschaft standen, in den Schoß des Mutterlandes führen sollten. Dies sei eine Frage der nationalen Ehre gewesen. Dementsprechend hätten die bulgarischen Soldaten im Gefühl der Pflichterfüllung für die Freiheit ihrer Nation die Hauptlast des Krieges getragen, während ihre Verbündeten - Serben und Griechen - vor allem Makedonien ohne größere Anstrengungen besetzt hätten. Der einzige Vorwurf, den Dimitrov in diesem Zusammenhang Zar Ferdinand macht, ist der diplomatischer Ungeschicklichkeit. Prinzipiell seien Serbien und Griechenland aufgrund ihrer Ansprüche in Makedonien, das bulgarisch besiedelt gewesen sei, 1912

11 Ebd. Zur vollständigen Unabhängigkeit: C. Todorova, Die europäische Diplomatie und die Erklärung der Unabhängigkeit Bulgariens im Jahre 1908, in: Bulgarisch-deutsche Beziehungen, Bd. 4, 127-152.

12 Vgl. z. B. G. Markov, Germanija i učastieto na Bălgarija v balkanskite vojni (1912-1913), in: Bulgarisch-deutsche Beziehungen, Bd. 4, 153-180. 
die falschen Verbündeten zu seiner Befreiung gewesen, und nach dem Ersten Balkankrieg hätte man die Spannungen zwischen beiden diplomatisch nützen müssen. Statt dessen habe Ferdinand zu kompromißlos auf Konfrontation zur Befriedigung seiner Ansprüche auf Makedonien gesetzt und dadurch die hinterhältige Strategie Rumäniens und der Türkei ermöglicht. Die Teilnahme am Ersten Weltkrieg sei dann als Kompensationsmaßnahme die logische Konsequenz aus dem verlorenen Zweiten Balkankrieg gewesen. Diesen Gesamtzusammenhang, die "nationalen Einigungskriege“, betrachtet Dimitrov zusammenfassend als legitimen Ausdruck des Strebens Ferdinands nach der Erfüllung seiner nationalen bulgarischen Mission zur Vereinigung aller vermeintlich ethnischen Bulgaren in einem Staate. Getrübt wird das Bild des Monarchen in dieser Geschichtsauffassung, die als charakteristisch für weite Teile der interessierten Öffentlichkeit gelten darf, also weniger durch eine prinzipielle Ablehnung seiner Politik und des Krieges als Mittel derselben, als vielmehr durch die Kritik an seinen „handwerklichen" Fehlern ${ }^{13}$.

Die stärkste Präsenz aller bulgarischen Monarchen im öffentlichen Geschichtsbewußtsein des Landes hat indes Zar Boris III. Seine Person wird am intensivsten mit dem Gedanken der Monarchie in Verbindung gebracht, d. h. die meisten Diskussionen um den Wert dieser Staatsform bewegen sich weniger auf einer allgemeinen bzw. abstrakten oder gar staatsphilosophischen Ebene; vielmehr wird vorwiegend anhand der konkreten Politik des Zaren erörtert, ob die Monarchie für Bulgarien Fluch oder Segen gewesen sei und - im Falle ihrer Wiedereinführung - erneut sein könnte.

Im Mittelpunkt des historischen Interesses an Boris stand sowohl vor als auch nach 1989 das letzte Drittel seiner Regierungszeit, also die Jahre von 1935 bis zu seinem unerwarteten Tode 1943. Während dieser Periode nahm der Zar in autoritärer Weise persönlich Einfluß auf die Politik und kann daher für alle wichtigen Entscheidungen direkt verantwortlich gemacht werden. Eine besondere Bedeutung erhält dieser Sachverhalt dadurch, daß in jene Jahre der Zweite Weltkrieg fiel, wodurch Bulgarien und damit Boris III. gezwungen war, grundlegende Entschlüsse nicht nur über die außenpolitische Orientierung, sondern auch über die innere Gestaltung des Landes zu treffen ${ }^{14}$.

Aufgrund der 1935 eingeführten monarchisch-autoritären Regierungsweise, für die sich der Zar in seiner Unzufriedenheit mit der ein Jahr zuvor durch einen Militärputsch an die Macht gekommenen „Zveno“-Regierung entschieden hatte,

13 B. Dimitrov, Bulgaria in the wars for national unification, in: >www.bulgaria.com/ history/wars.html<.

14 N. Poppetrov, Autoritarismus und Autoritäres Regime in Bulgarien, in: E. Oberländer, $R$ Jaworski u.a. (Hrsg.), Autoritäre Regime in Ostmitteleuropa 1919-1944 (Mainz 1995) 181-195, hier 195; M. Wien, Antisemitismus in Bulgarien im Spiegel der Parlamentsdebatten zum „Gesetz zum Schutze der Nation“ 1940 (Magisterarbeit München 1999); V. Migev, Formirane na koncepcijata za parlamentarnija model na bǎlgarskata monarchofašistka dăržava (1937-1938), in: Bălgarska Akademija na Naukite. Institut po istorija (Hrsg.), Obštestvenopolitičeskijat život na Bălgarija 1878-1944 (= Izsledvanija po bălgarska istorija 10, Sofia 1990) 289-326. 
sowie aufgrund des 1941 offiziell besiegelten Bündnisses mit Hitler-Deutschland, erhielt diese Periode der bulgarischen Geschichte von der sozialistischen Historiographie das unmißverständliche Etikett „monarcho-faschistisch“15. Dabei war es zumeist diese außenpolitische Nähe zu Deutschland in Verbindung mit einzelnen strukturellen Ähnlichkeiten im inneren Gefüge beider Staaten, die als argumentative Stütze für diese plakative Bezeichnung diente. Diese erleichterte es der bulgarischen Geschichtsschreibung vor 1989 wiederum, das Regiment des Zaren Boris in ihre ideologisch vorgegebenen Interpretationsmuster einzuordnen. Erst die Charakterisierung des Systems als „faschistisch“ lieferte das nötige Feindbild, das die oppositionelle Untergrundtätigkeit der kommunistisch dominierten „Vaterländischen Front" während des Krieges als "antifaschistisch " legitimierte ${ }^{16}$. Zugleich wurden in bisweilen paradoxer Weise die Momente, in denen sich das Regime als inkonsequent oder auch konziliant erwies, als Erfolge dieses Widerstandskampfes reklamiert, wodurch das Faschismus-Argument eine wohl eher ungewollte Aufweichung erfuhr. Denn ein Regime als „faschistisch“ und zugleich stellenweise „nachgiebig“ zu bezeichnen, erscheint wenn nicht als Paradoxie, so doch zumindest als Eingeständnis gravierender Unterschiede zu einem System wie dem deutschen Nationalsozialismus.

Einer dieser Momente der Nachgiebigkeit steht heute wie vor 1989 in der Diskussion um die bulgarische Monarchie unangefochten im Zentrum der Aufmerksamkeit: das Überleben der ca. 48000 bulgarischen Juden während des Zweiten Weltkrieges. Da über die positive Bewertung dieser Tatsache, die aufgrund des damaligen bulgarisch-deutschen Bündnisses vielfach Erstaunen hervorgerufen hat, ein Dissens kaum möglich ist, verlegte sich die Debatte von Anfang an auf die Frage, wer sich dieses Verdienst erworben hat, wobei vor 1989 ein freier Diskurs darüber praktisch nicht stattfinden konnte ${ }^{17}$. Die herrschende KP nahm die „Errettung" der Juden als Leistung für sich in Anspruch; unter ihrer Führung habe das bulgarische Volk den Zaren gezwungen, die bereits im Anlaufen befindlichen Deportationsmaßnahmen im März 1943 zu stoppen. Boris nimmt in diesem Szenario die Rolle des Erfüllungsgehilfen der deutschen Nationalsozialisten und der bulgarischen „monarchofaschistischen Oberschicht“ ein, die Kommunisten, deren Vorsitzender in Bulgarien damals Todor Živkov war, die Rolle der wahren Vertreter des Volkes und der Erretter der Juden ${ }^{18}$.

Bemerkenswert an der Entwicklung des Diskurses über das Schicksal der bulgarischen Juden während des Zweiten Weltkrieges ist, daß er auch nach 1989 nicht wesentlich differenzierter geführt wurde als zuvor. Im Zuge der allgemeinen Po-

15 Migev, Formirane; Poppetrov, Autoritarismus; Gelegentlich setzt die sozialistische Historiographie den Beginn der "monarcho-faschistischen " Periode bereits mit dem Sturz der Regierung Stambolijski im Jahre 1923 an. Vgl. Koen, Die monarchofaschistische.

16 Migev, Formirane.

17 Vgl. dazu I. Dimitrov, The Main Factor of the Salvation of the Bulgarian Jews, in: Jahrbuch der jüdischen Kulturorganisation in Bulgarien 24 (Sofia 1989) 242. Außerdem: $M$. Wien, Antisemitismus 1.

18 Ebd. 
larisierung des politischen Lebens wurde die Antwort auf die Frage nach dem Verdienst am Überleben der Juden zum Bekenntnis der Zugehörigkeit zu einem der beiden politischen Lager - dem "roten“, sozialistischen oder dem „blauen“ der oppositionellen Sammlungsbewegung „Union der demokratischen Kräfte“ (UDK). Die Angehörigen und Sympathisanten des blauen Lagers setzten der bisher gültigen These von der kommunistischen Führung im Kampf gegen die Deportation der Juden entgegen, daß es eigentlich Zar Boris selbst gewesen sei, der sie vor den Vernichtungslagern im deutsch besetzten Polen bewahrt habe ${ }^{19}$. Im Gefolge dieser Argumentation, die wiederum lediglich um die personalisierte Frage kreiste, wer der Retter gewesen sei, und nicht, welche Umstände und objektiven Faktoren diese Errettung ermöglichten, setzte eine umfassende Neu-Bewertung der Politik des Monarchen ein. Auch hier nahmen Journalismus, Publizistik und Populärwissenschaft die Vorreiterrolle ein, wobei es ohnehin scheint, daß die Grenzen zur „akademischen“ Geschichtswissenschaft während der 90er Jahre sowohl personell als auch qualitativ verschwammen ${ }^{20}$.

Boris wurde nun vom faschistischen Unterdrücker der Interessen des Volkes zum fürsorglichen Landesvater, der es nicht nur geschafft habe, „seine“ Juden vor deutschem Zugriff zu schützen, sondern auch, seinem Land die Teilnahme am Feldzug gegen die Sowjetunion zu ersparen. In der neuen Diktion erscheint der Monarch nicht mehr als gewissenloser Außenpolitiker, der das Bündnis mit den deutschen Gesinnungsgenossen unter Verrat der eigenen nationalen Belange gesucht habe, sondern vielmehr als gewiefter Taktiker, als „Fuchs“, wie er gelegentlich sogar betitelt wird, der aus der Kooperation mit NS-Deutschland angesichts ihrer Alternativlosigkeit das Beste gemacht habe ${ }^{21}$. Für Momente, die sein Bild als Retter der Juden eigentlich trüben müßten, sind relativ schnell entschuldigende Erklärungen gefunden worden. So wurde angesichts der Tatsache, daß im März 1943 aus den bulgarisch besetzten thrakischen und makedonischen Gebieten rund 11000 Juden in die Vernichtungslager deportiert wurden, geltend gemacht, daß diese Territorien zu diesem Zeitpunkt eben nur besetzt und bulgarisch verwaltet, aber nicht formell annektiert gewesen seien und daß demzufolge eigentlich nur die deutschen Behörden dort den Gang der Dinge bestimmt hätten. Zar Boris habe also gar nichts gegen die Deportation tun können ${ }^{22}$. Diese Argumentation geht indes darüber hinweg, daß sich das bulgarische „Kommissariat für Judenfragen“

19 Ebd.

20 Ebd. 1 f., 146. Zur These von der Rettung der Juden durch die Kommunisten vgl. das Jahrbuch der jüdischen Kulturorganisation in Bulgarien vor 1990. Die Auffassung, Zar Boris habe die Juden gerettet, fand vor allem nach der Wende des 10. November 1989 in Bulgarien Anklang. So zum Beispiel bei $H$. Bojadžiev, Spasjavaneto na bǎlgarskite evrei prez Vtorata svetovna vojna (Sofia 1991).

21 Ebd. Vgl. außerdem G. Nissim, Der Mann der Hitler stoppte (Berlin 2000). Zwar handelt es sich bei Nissim nicht um einen bulgarischen Autor, sondern um einen Italiener. Als das Buch aber 1998 in der bulgarischen Übersetzung erschien, wurde er in Sofia mit dem Orden „Reiter von Madara“ ausgezeichnet, der Ausländern verliehen wird, die sich um Bulgarien verdient gemacht haben.

22 Demokracija, Online-Ausgabe, 20. 7. 2000. 
unter Aleksandăr Belev und damit der Monarch als politisch Verantwortlicher zuvor mit den Vertretern des Reichssicherheitshauptamtes, an ihrer Spitze Theodor Dannecker, über die Modalitäten der Aktion geeinigt hatte und daß bulgarische Polizisten und Soldaten an ihrer Durchführung beteiligt waren. Die politische Führung Bulgariens muß also zumindest als Erfüllungsgehilfe für die Ermordung von 11000 thrakischen und makedonischen Juden betrachtet werden ${ }^{23}$.

In ähnlicher Weise wird versucht, den Zaren von der Verantwortung für die vorausgegangene antijüdische Gesetzgebung zu befreien. Das „Gesetz zum Schutze der Nation", das die Juden Bulgariens analog den Nürnberger Rassengesetzen aus den meisten Bereichen des öffentlichen Lebens ausschloß und vom Monarchen im Januar 1941 unterzeichnet und verkündet worden war, wird als reine Reaktion auf deutschen außenpolitischen Druck interpretiert, so als ob ohne seine Verabschiedung automatisch ein deutscher Einmarsch erfolgt wäre ${ }^{24}$.

Im Gegenzug wird die außenpolitische Geschicklichkeit des Zaren gelobt. In einer überwiegend pragmatischen Sichtweise, die rein erfolgsorientiert argumentiert, sich moralischer Wertungen weitgehend enthält und sich überdies die Maßstäbe des traditionellen bulgarischen Nationalgedankens zueigen macht, gelten nicht nur das Heraushalten Bulgariens aus dem Überfall auf die UdSSR, sondern auch die mit Hilfe Hitlers - wenn auch mit Ausnahme der Süd-Dobrudža nur vorübergehend - erreichte Gewinnung für Bulgarien reklamierter Gebiete als Aktiva in der Regierungsbilanz Boris' III. Seine Schläue, sein Lavieren gegenüber Deutschland, das sowohl in außenpolitischen Vorteilen als auch in der Verhinderung der Auslieferung von 48000 bulgarischen Juden resultierte, prägen in der Hauptsache das insgesamt positive Bild, das die heutige bulgarische Gesellschaft von ihrem eigentlich letzten Monarchen hat. Die zweifellos vorhandenen faschistoiden Elemente seines Regimes, die das Urteil der Kommunisten über ihn bestimmten, finden dagegen kaum mehr Beachtung 25.

Dabei geht es in diesem Zusammenhang nicht nur um eine Neubewertung der monarchischen Staatsform anhand ihrer wichtigsten Vertreter; als Motiv kommt außerdem die Suche nach einer nationalen Identifikationsfigur hinzu. Boris wird also auf diese Weise nicht nur zum personifizierten Beweis, daß eine Monarchie auch Gutes bewirken kann, er wird darüber hinaus zur Verkörperung des guten, vorsozialistischen Bulgarien schlechthin und damit auch zu einem historischen Ansatzpunkt für die „Rückkehr" des Landes in seine Geschichte nach 45 Jahren Sozialismus. Zur Aufrechterhaltung dieses Bildes, das in weiten Teilen der bulgarischen Öffentlichkeit zur Vorstellung von nationaler Würde gehört, ist es notwendig, die beschriebene Idealisierung der Person des Zaren zu einer unumstößlichen Gewissheit zu machen, zum Allgemeingut patriotischer Gesinnung. Im Sommer des Jahres 2000 war dieser Prozeß offenbar schon so weit fortgeschritten,

23 Wien, Antisemitismus 146-154.

24 Ebd. 29-33. Vgl. hierzu außerdem H.-J. Hoppe, Bulgarien - Hitlers eigenwilliger Verbündeter. Eine Fallstudie zur nationalsozialistischen Südosteuropapolitik (Stuttgart 1979).

${ }_{25}$ Poppetrov, Autoritarismus und B. Dimitrov, Bulgaria in the interim between bourgeois democracy and fascism, in: >www.bulgaria.com/history/bulgaria/interim.html<. 
daß das Bild, das die Öffentlichkeit von Boris III. hatte, politisch entscheidungsrelevant geworden war. Denn zu dieser Zeit wurde in der israelischen HolocaustGedenkstätte Yad Vashem beschlossen, die Tafel, die im „Wald der Gerechten“ den Zaren als Retter der bulgarischen Juden ehrte, zu entfernen und durch eine allgemeinere, der Nation als ganzer gewidmeten zu ersetzen. Ein Sturm der Entrüstung brach in der politischen Öffentlichkeit, und zwar vor allem im „blauen“ Lager, los. Man faßte die Entfernung der Tafel so auf, als würde nun Bulgarien insgesamt das Verdienst der Errettung bestritten. Die Empörung steigerte sich noch, als der sozialistische Vizeparlamentspräsident Blagovest Sendov seine Zustimmung zu dieser Aktion verkündete. Hierauf warf man ihm, vor allem auch aufgrund seiner Parteizugehörigkeit, eine anti-bulgarische Haltung vor und forderte ihn zum Rücktritt auf ${ }^{26}$. Der Sofioter Bürgermeister Stefan Sofijanski erklärte sich unterdessen bereit, die Tafel von Yad Vashem zu übernehmen und in seiner Stadt an exponierter Stelle anzubringen ${ }^{27}$. Die Identifikation mit Boris III. war also in Bulgarien schon so weit fortgeschritten, daß man das Gedenken an ihn mit der Ehre der ganzen Nation verknüpfte.

Andererseits hat dies nicht verhindert, daß seine Epoche, und hier besonders die Jahre nach 1935, vielfach nach wie vor als die Periode des "Faschismus" bezeichnet wird, wobei dieser Begriff kaum mit konkreten Inhalten gefüllt ist. In entsprechender Weise werden die an der "Vaterländischen Front" und am Partisanenkampf beteiligten Kräfte als „antifaschistisch“ betitelt ${ }^{28}$. Hier zeigt sich beispielhaft das Nebeneinander in gegenseitigem Widerspruch stehender Geschichtsbilder in den Köpfen weiter Teile der bulgarischen Gesellschaft. Während auf der einen Seite inzwischen Bewunderung weckt, wie Zar Boris als verantwortungsbewußter Staatslenker sein Land durch die aufgepeitschte See des damaligen Weltgeschehens steuerte und seine autoritäre Innenpolitik die chaotische Parteienlandschaft beseitigte, bleiben auf der anderen Seite die in den Jahrzehnten der kommunistischen Diktatur erlernten Epochenbezeichnungen und klassenkämpferischen Klischeevorstellungen im historischen Bewußtsein vieler Menschen präsent ${ }^{29}$. Zwar ist anzunehmen, daß diese sich mit dem Heranwachsen neuer Generationen abschleifen und daß differenziertere und schlüssigere Geschichtsbilder die Oberhand gewinnen werden, eine wichtige Voraussetzung hierfür wäre allerdings auch die Überwindung der extremen Bipolarität der politischen Landschaft Bulgariens, die unter anderem eine sachlichere Auseinandersetzung über die Zeit des Sozialismus, die frei von ideologischen Kampfbegriffen wie "Faschismus“ oder „Antifaschismus" sein müßte, ermöglichen würde. Ob die Parlamentswahlen vom Juni 2001 mit dem Sieg Simeons II., die vielfach als das Ende des bisherigen Parteien-

26 Demokracija, Online-Ausgabe, 20. 7. 2000.

27 Ebd.

28 B. Dimitrov, Bulgaria in the interim. Beispiele für differenziertere Darstellungen, die den Begriff "Faschismus" vermeiden, sind etwa: E Kalinova, $I$. Baeva, Bălgarskite prechodi 1944-1999 (Sofia 2000) 13 und P. S. Cvetkov, Demokracijata i nejnite alternativi v Bălgarija mežu dvete svetovni vojni, in: 120 godini 177-187.

29 Ebd. 
systems interpretiert worden sind, ein Schritt in diese Richtung waren, muß sich beim nächsten Urnengang erst noch bestätigen. Dagegen spricht jedenfalls, daß Untersuchungen den Sieg Simeons weniger auf umwälzende Veränderungen bei den Parteibindungen in der Bevölkerung zurückführen, sondern vielmehr auf die geringe Mobilisierung der Stammwählerschaft der etablierten Parteien ${ }^{30}$.

Ansätze zu einer ausgewogenen Betrachtung der Regierungszeit Boris' III., die sich abseits der tagespolitischen Konfrontationen entwickelt haben, sind bisher kaum über die engen Zirkel des akademischen Diskurses hinausgedrungen. Hervorzuheben sind in diesem Zusammenhang beispielsweise Autoren wie Nikolaj Poppetrov oder Evgenija Kalinova und Iskra Baeva, die sowohl die autoritären Züge der Herrschaft des Zaren als auch die Unterschiede zu faschistoiden Regimes der selben Zeit klar herausgearbeitet haben ${ }^{31}$. Besonders Poppetrov, der entsprechende Aufsätze bereits in den 80er Jahren in westdeutschen Fachzeitschriften veröffentlichte, hat eine klare und zugleich differenzierte Charakteristik des Boris'schen Regierungsstils von seiner Thronbesteigung 1918 bis 1943 geboten. Anstelle des ideologisch besetzten Begriffes „Monarchofaschismus“ stuft er die Regierung des Zaren als „monarchisch-autoritär mit einzelnen Elementen der Faschisierung“ ein. Besonderen Wert legt er auf die Feststellung, daß die Regierungszeit des Monarchen durch eine Reihe von diesem selbst begangener Rechtsbrüche gekennzeichnet ist. Boris III. habe von Anfang an nur wenig Respekt vor der bulgarischen Verfassung gezeigt und sich nicht selten über sie hinweggesetzt. Und tatsächlich wies bereits sein Amtsantritt diesen Makel auf. Laut Verfassung wäre er nämlich verpflichtet gewesen, vor einer sogenannten Großen Volksversammlung einen Eid auf eben dieses Staatsgrundgesetz abzulegen. Boris unterließ es einfach. Den eklatantesten Verfassungsbruch beging er indes 1935 mit dem Verbot aller Parteien und der auf unbestimmte Zeit erfolgten Auflösung der Volksversammlung, wodurch er die bulgarische parlamentarische Demokratie zunächst und zumindest der Form nach in eine Königsdiktatur verwandelte ${ }^{32}$.

\section{König und Ministerpräsident in einer Person}

Gerade dieses Durchgreifen ist es indes, was sich heute die meisten Sympathisanten der Monarchie in Bulgarien zumindest unterschwellig von dieser Staatsform erwarten. Durch die Beschwörung des Bildes des Zaren Boris III., der ihrer Ansicht nach eine kluge Außenpolitik führte, mit innenpolitischem Gezänk Schluß machte und zugleich durch die Rettung der Juden Menschlichkeit bewies, verleihen auch heute weite Teile der Bevölkerung ihrem Unmut über die offensichtliche Unfähigkeit der bulgarischen Politik, gleich welcher Couleur, Ausdruck. Diese Disposition der Wählerschaft war es, die dem Sohn Boris', Simeon II., dem for-

$30 \mathrm{Vgl}$. Wien, Parlamentswahlen.

31 Vgl. Poppetrov, Autoritarismus und Kalinova, Baeva, Bălgarskite prechodi.

32 Poppetrov, Autoritarismus 195. 


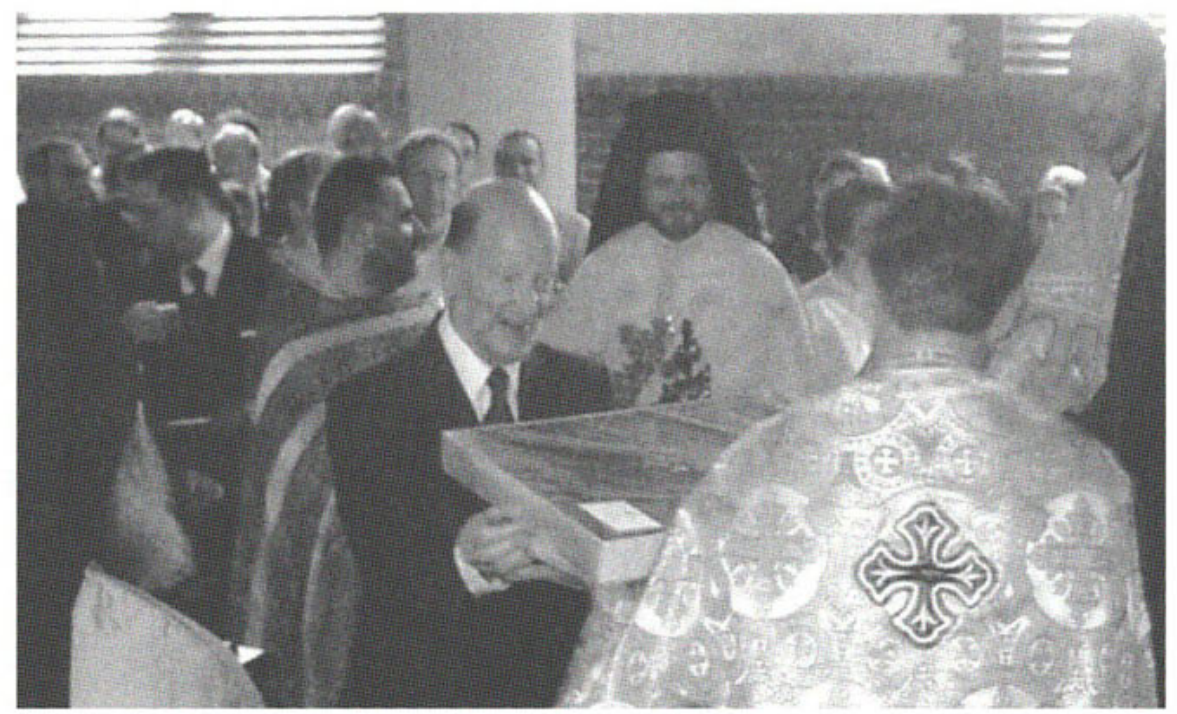

Copyright: http://www.osteuropa-zentrum.de

mell letzten Zaren, im Jahre 2001 die Chance eröffnete, die politische Bühne Bulgariens zu betreten. Zwar wiesen Umfragen aus, daß es keine Mehrheit in der Bevölkerung für die Wiedereinführung der Monarchie gab; ohne die beschriebenen Änderungen im diesbezüglichen Geschichtsbild seit 1989 wäre aber die große Sympathie, die Simeon überall entgegengebracht wurde, wo er seit 1996 in Bulgarien auftrat, kaum zu erklären gewesen ${ }^{33}$. Der Glanz der durch seinen Vater verkörperten Monarchie strahlte auch auf ihn ab und führte, vor allem in ländlichen Regionen, bisweilen dazu, daß die Sympathien sich in eine regelrechte Vergötterung steigerten, also religiöse Züge annahmen. Hierdurch wurde klar, was sich die Menschen von Simeon erhofften: Ähnlich seinem Vater sollte er beinahe nach Art eines Heilsbringers "die Zügel in die Hand nehmen" und jenseits der Niederungen des politischen Tagesgeschäfts die Lösung der Probleme, die in Bulgarien in erster Linie sozialer Natur sind, bringen. Deutlich spürbar war, daß hinter diesen Wunschvorstellungen auch das Bedürfnis steckte, von gesellschaftlicher Verantwortung befreit zu werden und die Segnungen der „königlichen“ Politik nur noch empfangen zu müssen. Der Politik als solcher sollte gewissermaßen der politische Charakter genommen werden ${ }^{34}$.

Diese Erwartungen waren sicherlich nicht Ausdruck tiefsitzender antidemokratischer Überzeugungen im bulgarischen Volk, wohl zeigten sie aber große Enttäuschung über die seit 1989 in der parlamentarischen Demokratie erbrachten politischen Leistungen. Da weder die Sozialisten noch die seit 1997 regierenden „Demokratischen Kräfte" eine nennenswerte Steigerung des allgemeinen Lebens-

33 Wien, Parlamentswahlen 13-15.

34 Ebd. 
standards hatten herbeiführen können und sich überdies durch zahllose Fälle von Korruption und Bereicherung diskreditiert hatten, hatte Simeon, als er im Frühjahr 2001 in den Wahlkampf einstieg, einen doppelten Vorteil: Zum einen umgab ihn der Glorienschein eines Königs, zum anderen kam er von „außen“, d.h. aus dem spanischen Exil, war also durch keinerlei Machenschaften der bulgarischen Politik kompromittiert. Dieses hauptsächliche Unterscheidungsmerkmal im Verhältnis zur etablierten bulgarischen Politik war der wichtigste Hebel, den Simeon im Streben nach einem Wahlerfolg ansetzte. Im Wissen um den schlechten Ruf der meisten Politiker warb Simeon mit der eingängigen Parole: „Aufrichtigkeit in allem! "35

Sein überwältigender Wahlsieg ist indes sicher nicht allein auf seinen Ehrlichkeitsanspruch und die ihn umgebende königliche Aura zurückzuführen, sondern zweifellos auch auf die vielen handfesten Versprechen und programmatischen Aussagen, die Simeon in seinem Wahlprogramm machte. Die bemerkenswerteste war die Frist, die er sich selbst zu einer deutlich spürbaren Anhebung des Lebensstandards der breiten bulgarischen Bevölkerung setzte: Er wollte dieses Ziel innerhalb von 800 Tagen nach Beginn der Legislaturperiode erreicht haben, wobei zum Zeitpunkt der Verkündung des Programms noch nicht klar war, ob Simeon selbst im Falle eines Wahlsieges Regierungschef werden, wer also für die Umsetzung seines Programms verantwortlich sein würde. Herausragend war außerdem die Ankündigung, daß es günstige staatliche Kredite zur Förderung mittelständischer Unternehmen geben würde, um die Dominanz einiger mit mafiösen Struk-

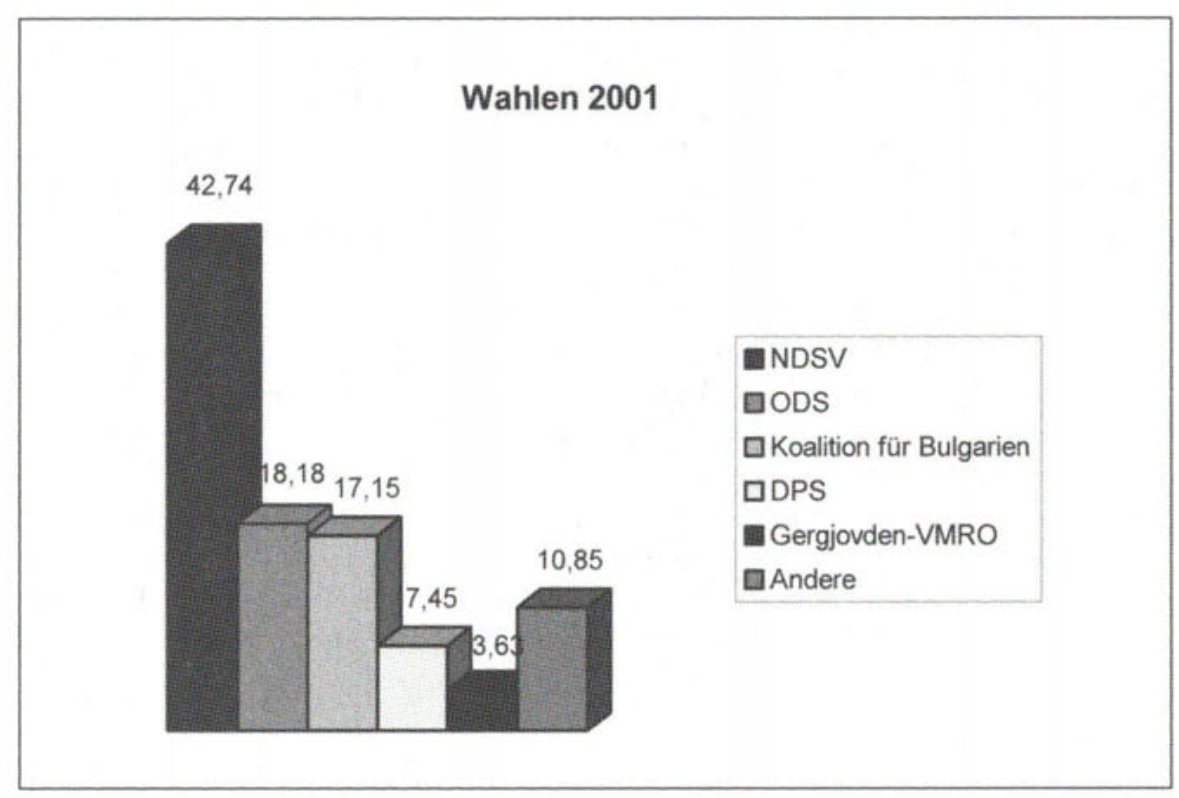

35 Ebd. 30. 
turen durchsetzter Großfirmen zu durchbrechen und Arbeitsplätze zu schaffen. Gleichzeitig sollten in allen Bereichen die Steuern signifikant gesenkt werden. Durch diese und andere Punkte nahm das Programm Simeons einen derart umfassenden Charakter an, daß seine komplette Umsetzung tatsächlich nichts weniger als eine völlige Neugestaltung der sozioökonomischen Verhältnisse Bulgariens bedeutet hätte ${ }^{36}$.

Abgesehen von der Frage seiner praktischen Durchführbarkeit ist es allerdings kaum mit Sicherheit zu bemessen, welchen Stellenwert das Programm bei der Entscheidung der Wähler, Simeon ihre Stimme zu geben, eingenommen hat. Angesichts der offensichtlichen Emotionalisierung des Wahlkampfes darf angenommen werden, daß es entsprechend emotional bewegende Momente waren, die den Ausschlag gegeben haben. $\mathrm{Zu}$ diesen zählten weniger die Feinheiten des Wahlprogramms, als vielmehr dessen markanteste Punkte sowie die Abneigung gegen die bekannten Politiker, in erster Linie aber die Sympathie für die alles überstrahlende Person Simeons.

Hierin liegt indes ein Schlüssel zur Einschätzung der Bedeutung, die dem Bild, das sich die Bulgaren von der Staatsform der Monarchie machen, sowohl in ihrem historischen als auch in ihrem politischen Bewußtsein beizumessen ist. Denn der außerordentlich hohe Grad an Personalisierung des Wahlkampfes gab in Verbindung mit der Frage, inwieweit sich Simeon selbst als "Zar" präsentierte, Aufschluß über die Attraktivität des monarchischen Gedankens für das Wahlvolk:

Simeon setzte - mit Erfolg, wie das Ergebnis zeigte - sehr klar auf seinen königlichen Status. Dies wurde auf vielfältige Weise deutlich: Zuvorderst ist hier die Namensgebung für sein Wahlbündnis zu nennen, das im April 2001 aus zwei Splitterparteien gebildet worden war, nachdem es Simeon gerichtlich verwehrt worden war, mit einer eigenen Partei anzutreten. Das Bündnis erhielt den Namen „Nationale Bewegung Simeon der Zweite“ ${ }^{37}$. Hierdurch wurde unmißverständlich kundgegeben, daß der Ex-Zar nicht nur die zentrale, sondern auch die konstituierende Gestalt, mithin also die personifizierte Existenzberechtigung der Bewegung war. Mit der Verwendung seines Herrschernamens unter Einschluß der königlichen „Nummerierung“ anstelle des bürgerlichen bulgarischen „Sakskoburggotski" wurde außerdem der Eindruck erweckt, Simeon erhebe nach wie vor Anspruch auf den Thron. Dies verstärkte sich dadurch, daß, wie der Name seines Bündnisses suggerierte, die Person Simeons „des Zweiten“ zugleich Programm war. Da sich der Namensgeber außerdem von seinen Vertrauten und Mitarbeitern als „Seine Majestät" betiteln ließ und in symbolträchtiger Art und Weise seinen Wohnsitz in dem an ihn zurückgegebenen alten königlichen Landschloß „Vranja“ genommen hatte, konnte es kaum verwundern, daß vielerorts gemutmaßt wurde, er strebe letztlich die Wiedereinführung der Monarchie und seine Rückkehr auf

36 Vgl. Bălgarija - dobăr dom za svoite graždani. Predizborna program na koalicija „Nacionalno dviženie Simeon Vtori““ (Sofia 2001).

37 Ebd. 
den Thron an ${ }^{38}$. Genährt wurden diese Gerüchte indes nicht nur durch die Benennung seines Wahlbündnisses und dadurch, daß er weiterhin einige seiner herrscherlichen Attribute führte, sondern auch durch zweideutige Äußerungen von seiner Seite und durch unklares Verhalten.

Wie nicht anders zu erwarten, gehörte zu den ersten und am häufigsten von Journalisten an Simeon gerichteten Fragen eben diejenige nach der Wiederherstellung der bulgarischen Monarchie. Der Tenor seiner Antworten bestand bis kurz vor der Wahl darin, daß sich diese Frage „im Moment “ nicht stelle bzw. daß sie zur Entscheidung anstünde, sobald die Zeit dafür reif sei. Erst unmittelbar vor dem Urnengang dementierte Simeon deutlicher seine Ambitionen auf den Thron, wollte aber die Wiedereinführung der Monarchie immer noch nicht für alle $\mathrm{Zu}$ kunft ausschließen. Dies wurde so aufgefaßt, daß er möglicherweise nicht für sich selbst, dafür aber für seinen ältesten Sohn, Kronprinz Kardam, und seine Dynastie insgesamt die Möglichkeit schaffen wollte, erneut an die Spitze des Staates zu treten ${ }^{39}$. Weiterhin wurde spekuliert, er würde zunächst, d.h. im Herbst 2001 bei den bulgarischen Präsidentschaftswahlen, antreten, um dann als Staatsoberhaupt mit Hilfe der von seiner Bewegung zuvor errungenen parlamentarischen Mehrheit eine Verfassungsänderung im monarchischen Sinne durchzusetzen. Zwar wäre ein solches Vorhaben von vornherein zum Scheitern verurteilt gewesen, da ein Präsidentschaftskandidat zum Zeitpunkt der Wahl seit mindestens fünf Jahren seinen Wohnsitz in Bulgarien haben muß $3^{40}$, was auf Simeon nicht zutraf; dies tat jedoch den Spekulationen keinen Abbruch, es gehe ihm nicht um die auf einem Regierungschef lastende politische Verantwortung, sondern vielmehr um die landesväterliche Rolle des gekrönten Staatsoberhauptes. Unmittelbar nach dem für ihn triumphalen Urnengang schien Simeon diese Spekulationen zu bestätigen. In einer für eine parlamentarische Demokratie äußerst ungewöhnlichen Weise ließ er die Öffentlichkeit ungefähr drei Wochen lang warten, bevor er sich dazu durchrang, seine Bereitschaft zur Übernahme des Amtes des Ministerpräsidenten zu verkünden. Er erweckte dabei allgemein den Eindruck, als tue er dies nur widerwillig und beuge sich nach langem Zögern dem Zwang, als Führer seiner Bewegung Verantwortung übernehmen zu müssen. Wie es schien, hatte er diese Entwicklung nicht vorhergesehen und den Posten des Regierungschefs eigentlich als für seine Person unangemessen betrachtet. Denn nun bestand die Gefahr, daß die Widrigkeiten des politischen Tagesgeschäfts nicht nur ihn selbst, sondern auch die Idee der Monarchie, die er mit seiner Person in der öffentlichen Wahrnehmung eng verknüpft hatte, dauerhaft beschädigen könnten ${ }^{41}$.

Unabhängig vom Erfolg oder Mißerfolg seiner sich anschließenden Regierungstätigkeit hatte die Wahl jedoch klargemacht, daß königliche Ausstrahlung,

38 Wien, Parlamentswahlen 27. Außerdem: F. Nienbusen, Im Reich des schüchternen Königs, in: Süddeutsche Zeitung vom 16. 7. 2001, 10.

39 Ebd.

40 Konstitucija na Republika Bălgarija, 13.7. 1991, čl. 93 (2). Vgl. >www.parliament.bg/ const.html<, 7. 5. 2001.

41 Wien, Parlamentswahlen $31 \mathrm{f}$. 
wie sie Simeon besaß, eine große Faszination auf die bulgarische Wählerschaft ausüben und sich damit auch in Stimmen niederschlagen konnte. Wie sich dieser monarchische Bonus aber konkret auf das Wahlverhalten auswirkte bzw. wie er die Wähler zum Votum für Simeon motivierte, ist nur sehr schwer zu bestimmen. Denn ein klares politisches Programm kam durch diesen Faktor nicht zum Ausdruck. Durch die Unsicherheit und die Spekulationen bezüglich einer Wiedereinführung der Monarchie kam es vielmehr zu der widersprüchlichen Situation, daß sich ein Faktor auf die Stimmabgabe auswirkte, dessen Realitätsgehalt kaum klar zu erkennen war. Die Suche nach den Vorstellungen und Erwartungen, die die Bürger mit der Person des „Zaren“ Simeon II. verbanden, konnte dementsprechend nur ein diffuses Bild ergeben. Diffus waren allerdings auch die Vorstellungen der meisten Bulgaren von dem, was eine Monarchie als Staatsform überhaupt bedeutet ${ }^{42}$. Diese Vielschichtigkeit deutet zugleich darauf hin, daß Simeon aus den unterschiedlichsten Gründen gewählt worden ist. Jeder nahm sich gewissermaßen das als Motiv, was ihm persönlich an der Monarchie oder zumindest der monarchischen Ausstrahlung des Kandidaten am besten gefiel. Daher läßt sich aus dem Wahlausgang auch nicht ohne weiteres schließen, daß die Wahl Simeons ein Votum für dessen Reinthronisierung gewesen sei. Wie bereits angedeutet, hatten Umfragen keine Mehrheit für einen derartigen Schritt ergeben. Trotzdem aber ließen sich die Wähler durch die zumindest nicht von der Hand zu weisenden Spekulationen, die in diese Richtung deuteten, nicht abschrecken. Eine eindeutige Ablehnung der Monarchie war aus ihrem Verhalten ebenfalls nicht abzulesen.

Aufgrund der unklaren Vorstellungen vom Wahlprogramm der Bewegung Simeons und von der verfassungsrechtlichen Bedeutung der Monarchie ist allerdings anzunehmen, daß man sich in erster Linie auf die Person konzentrierte und seine Einstellung zu dieser Frage an ihr ausrichtete. Ähnlich verhielt es sich auch mit der Wandlung des allgemeinen historischen Bildes von der Monarchie vor 1944. Auch hier steht, wie bereits erörtert, die Person Boris' III. im Zentrum aller Erörterungen. Von der Achtung, die ihm im Verlauf der 90er Jahre zunehmend in Bulgarien entgegengebracht wurde, profitierte auch sein Sohn Simeon. Vielfach wurde in bisweilen naiv anmutender Weise die direkte Vererbbarkeit politischer Fertigkeiten angenommen und hieraus der Schluß gezogen, man habe in Simeon eine Kopie seines Vaters vor sich, die ähnlich wie der "Fuchs" in der Lage sei, Bulgarien durch die Unsicherheiten der kommenden Jahre hindurch zu steuern ${ }^{43}$. Die Ausschließlichkeit, mit der seine Person im Wahlkampf eingesetzt wurde, läßt sich dadurch veranschaulichen, daß er der einzige Repräsentant seiner Bewegung war, der auf Plakaten abgebildet wurde, wobei die Tagespresse den Effekt dieser Strategie noch verstärkte, indem sie ihn in den letzten Wochen vor der Wahl fast täglich auf den Titelseiten zeigte ${ }^{44}$.

42 Ebd. 13-15.

43 Ebd.

${ }_{44}$ Nienbusen, Im Reich. 


\section{Das personalisierte Monarchiebild als Mittel zur ideologisch- politischen Standortbestimmung}

An der Art der Wandlung des Geschichtsbildes, das die Bulgaren bezüglich ihrer zwischen 1878 und 1946 bestehenden Monarchie haben, zeigt sich, daß diese sehr stark, wenn nicht sogar hauptsächlich, in einer von politischen und sozialen Interessen und Entwicklungen angetriebenen Veränderung der Bewertung ihrer Hauptrepräsentanten bestand. Das Spektrum, innerhalb dessen sich diese Entwicklung vollzog, reichte von der vor 1989 obligatorischen prinzipiellen Ablehnung der Monarchie als „faschistisch“, wobei hier eigentlich nur Boris III. gemeint war, bis zu einer Situation zur Jahrtausendwende, in der offen über die Möglichkeit einer Wiedereinführung der Monarchie diskutiert wurde. Dies war dann in erster Linie mit dem Sohn Boris' III., Simeon II., assoziiert. Wie sehr das Verhältnis der politischen Kräfte zur Monarchie von ihrer Interessenlage und daraus resultierenden Wahlkampfstrategien bestimmt war, zeigte sich am deutlichsten an der Union der demokratischen Kräfte. Bis zum Ende der 90er Jahre war ihre zwar nicht monarchistische, aber doch monarchiefreundliche Haltung ein Ausdruck der Ablehnung des sozialistischen Regimes und der Abgrenzung von seinen postkommunistischen Nachfahren. Vertreter der Partei, wie etwa Ministerpräsident Kostov oder Staatspräsident Stojanov zeigten sich gerne mit Simeon in der Öffentlichkeit, seitdem dieser ab 1996 regelmäßig Bulgarien besuchte $e^{45}$. Wie bereits erwähnt, setzten sie auch die Wiederverwendung des königlichen Staatswappens durch. Als Simeon im Frühjahr 2001 unversehens zum politischen Gegner avancierte, brach diese Hoffierung unvermittelt $a b$, und man versuchte, den Gegenkandidaten nun auf die Statur eines Oppositionspolitikers mit hochstaplerischen Ambitionen zurechtzustutzen. Aus dem "Zaren“, der lebenden Reliquie des alten königlichen Bulgarien, sollte ein Parvenue werden, dem nicht zu trauen sei. Daß diese Strategie nicht verfing, zeigte das Wahlergebnis ${ }^{46}$.

Die anderen Parteien, für deren Machtposition die NDSV keine so große Bedrohung darstellte, hatten sich im Umgang mit Simeon unbefangener gezeigt. Die Sozialisten betrachteten ihn zwar nicht mehr wie vor 1989 als unerwünschte Person im Lande, behandelten ihn aber konsequent als normalen bulgarischen Staatsbürger, zeigten damit also zumindest eine Art von Nicht-Verhältnis zu seiner monarchischen Vergangenheit. Die Partei „Bewegung für Rechte und Freiheiten“, die die türkische Minderheit vertritt, ging mit Simeon eine Koalition ein, zeigte also keine Berührungsängste, da sie in ihrer Rolle als Klientelpartei nicht befürchten mußte, in den Verdacht des Monarchismus zu geraten ${ }^{47}$.

45 J. Gruber, Bulgarien im ersten Jahr der Regierung von Ex-König Simeon Sakskoburggotski. Handwerkliche Fehler des Kabinetts oder Beginn einer krisenhaften Entwicklung?, in: www.kas.de/publikationen/2002/ai/03_gruber.pdf, S 2.

46 Wien, Parlamentswahlen 13.

47 Ebd. $29 f$. 
Klar wurde durch die Ereignisse um die Wahl Simeons indes, daß die Einstellung der Bulgaren zur Monarchie und ihr diesbezügliches historisches Bewußtsein politisch konjunkturabhängig sind. Nach dem ersten Jahr seiner Zeit als Ministerpräsident deutet vieles darauf hin, daß die Begeisterung für ihn und damit auch die Monarchie im Abnehmen begriffen ist, wobei dies noch aus größerem zeitlichen Abstand zu überprüfen wäre. Die Woge der Sympathie, auf der er zuvor geschwommen war, war allerdings von zwei Faktoren getragen worden: der sozialen Notlage der Bevölkerung und ihrer politischen Ratlosigkeit einerseits und andererseits der Tatsache, daß mit Simeon ein Mann im richtigen Alter bereitstand, der den monarchischen Gedanken glaubhaft verkörpern konnte und als Politiker zumindest vorstellbar war. Dies erklärt auch, warum er der bisher einzige ehemalige osteuropäische Monarch ist, der in seinem Land nach den Jahrzehnten kommunistischer Diktatur wieder zu nennenswertem Einfluß gelangen konnte ${ }^{48}$.

48 Vgl. hierzu: H.- J. Hoppe, Saison für Könige! Macht das bulgarische Modell Schule?, in: Südosteuropa Mitteilungen 3 (2002) 54 67. 